\title{
Surgery for mesothelioma: Less is more, more or less
}

\author{
Steven Milman, MD, and Thomas Ng, MD
}

\author{
From the Department of Surgery, The Warren Alpert Medical School of Brown University, Providence, RI \\ Disclosures: Authors have nothing to disclose with regard to commercial support. \\ Received for publication Oct 31, 2017; accepted for publication Nov 10, 2017; available ahead of print Dec 6, \\ 2017. \\ Address for reprints: Thomas Ng, MD, 2 Dudley St, Suite 470, Providence, RI 02905 (E-mail: tng @ usasurg.org). \\ J Thorac Cardiovasc Surg 2018;155:1867-8 \\ $0022-5223 / \$ 36.00$ \\ Copyright (c) 2017 by The American Association for Thoracic Surgery \\ https://doi.org/10.1016/j.jtcvs.2017.11.029
}

Mesothelioma continues to be a devastating malignancy, with poor survival rates despite treatment. Surgery rarely achieves complete microscopic resection, and response with other modes of therapy is poor. In an attempt to improve survival rates, current treatment involves delivering several modalities of treatment in combination. In the setting of multimodality treatment, the extent of surgery has been debated, with most recent studies supporting less radical surgery of pleurectomy decortication (PD) in preference to extrapleural pneumonectomy (EPP). The article by Ambrogi and colleagues ${ }^{1}$ in this issue of the Journal reports one such study.

Ambrogi and colleagues ${ }^{1}$ present their experience of 49 patients with epithelial or biphasic mesothelioma who underwent PD (sparing diaphragm and pericardium) and hyperthermic intrathoracic chemotherapy, followed by adjuvant chemotherapy, at a single institution during a 10year period. The main objective was to assess feasibility, finding no 90-day mortality and an overall morbidity rate of $47 \%$. Secondary outcome assessed was survival, with median overall survival found to be 22 months (35 months for stages I and II, 17 months for stages III and IV) and 5year overall and disease-free survivals found to be $10 \%$ and $18.5 \%$, respectively.

Ambrogi and colleagues ${ }^{1}$ are to be congratulated on their good outcomes, specifically regarding the zero 90-day mortality and the very respectable survival rates. Before accepting this as the superior regimen for the treatment of mesothelioma, however, a more careful examination of the study's weaknesses is required. First, the number of patients evaluated is small. Second, the study duration is long, and significant advances in staging techniques and treatment are sure to occur with time. Third, the study lacks data on quality of life, which is an important aspect to consider when comparing different treatment regimens for aggressive malignancies. Finally, the study is retrospective and single cohort in nature. It therefore lacks control of important factors that can significantly affect outcomes, such as the selection of patients, the staging techniques used, the quality of surgery performed, and the delivery of postoperative therapy. With these limitations, meaningful

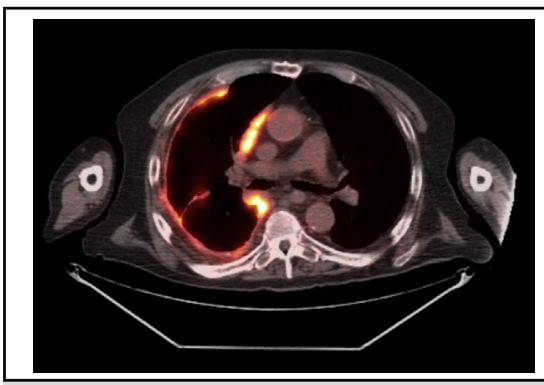

Positron emission tomographic scan: mesothelioma

Central Message

Current data increasingly support less radical surgery in the multimodality treatment of mesothelioma; however, a higher level of evidence is needed to confirm this.

See Article page 1857.

comparisons between this study of Ambrogi and colleagues ${ }^{1}$ and other published studies become difficult.

Despite these limitations, this study by Ambrogi and colleagues ${ }^{1}$ does have importance. Because long-term outcomes are poor, any treatment offered to patients with mesothelioma should, first and foremost, do no harm and also provide a measurable benefit; the zero 90-day mortality and the respectable survivals presented by Ambrogi and colleagues ${ }^{1}$ clearly show their treatment regimen of PD with intrathoracic hyperthermic chemotherapy to be in keeping with these criteria. Recent meta-analyses by Cao and colleagues ${ }^{2}$ and Taioli and colleagues ${ }^{3}$ found EPP to have higher mortality and similar overall survival when compared with PD. Because of the lack of randomized studies examined, however, these meta-analyses suffer from many of the limitations seen in the study by Ambrogi and colleauges, ${ }^{1}$ as discussed previously. Even with the publication of the only randomized trial comparing PD with EPP, by the Mesothelioma and Radical Surgery trial group, ${ }^{4}$ no firm conclusion regarding superiority can be made because of the significant limitations of the study, as pointed out by Rusch and colleagues. ${ }^{5}$

Even in the absence of a well-conducted randomized trial, it seems clear that PD results in less morbidity and mortality than EPP. It also seems clear that achieving complete microscopic resection is very unlikely, regardless of the surgical procedure performed, and for this reason combing surgery with other modes of therapy is essential. It 
would therefore seem reasonable that if complete macroscopic resection can be achieved by PD, then this less morbid procedure should be considered first, in preference to EPP. Until high-level data are available to indicate otherwise, however, EPP should not be entirely abandoned. Circumstances may arise warranting its consideration, such as a young healthy patient with extensive visceral pleural and parenchymal disease, for which complete macroscopic resection can only be achieved by pneumonectomy.

\section{References}

1. Ambrogi MC, Bertoglio P, Aprile V, Chella A, Korasidis S, Fontanini G, et al. Diaphragm and lung-preserving surgery with hyperthermic chemotherapy for malig- nant pleural mesothelioma: a 10-year experience. J Thorac Cardiovasc Surg. 2018;155:1857-66.e2.

2. Cao C, Tian D, Park J, Allan J, Pataky KA, Yan TD. A systematic review and metaanalysis of surgical treatments for malignant pleural mesothelioma. Lung Cancer. 2014;83:240-5.

3. Taioli E, Wolf AS, Flores RM. Meta-analysis of survival after pleurectomy decortication versus extrapleural pneumonectomy in mesothelioma. Ann Thorac Surg. 2015;99:472-80.

4. Treasure T, Lang-Lazdunski L, Waller D, Bliss JM, Tan C, Entwisle J, et al; MARS trialists. Extra-pleural pneumonectomy versus no extra-pleural pneumonectomy for patients with malignant pleural mesothelioma: clinical outcomes of the Mesothelioma and Radical Surgery (MARS) randomised feasibility study. Lancet Oncol. 2011;12:763-72.

5. Rusch V, Baldini EH, Bueno R, De Perrot M, Flores R, Hasegawa S, et al; Participants in 2012 International Mesothelioma Interest Group Congress. The role of surgical cytoreduction in the treatment of malignant pleural mesothelioma: meeting summary of the International Mesothelioma Interest Group Congress, September 11-14, 2012, Boston, Mass. J Thorac Cardiovasc Surg. 2013;145:909-10. 